\title{
Conditional Well-Posedness for an Inverse Source Problem in the Diffusion Equation Using the Variational Adjoint Method
}

\author{
Chunlong Sun, ${ }^{1,2}$ Qian Liu, ${ }^{1}$ and Gongsheng $\mathrm{Li}^{1}$ \\ ${ }^{1}$ School of Science, Shandong University of Technology, Zibo 255049, China \\ ${ }^{2}$ Department of Mathematics, Southeast University, Nanjing 210096, China \\ Correspondence should be addressed to Gongsheng Li; ligs@sdut.edu.cn
}

Received 22 March 2017; Accepted 31 May 2017; Published 27 June 2017

Academic Editor: Mikhail Panfilov

Copyright (C) 2017 Chunlong Sun et al. This is an open access article distributed under the Creative Commons Attribution License, which permits unrestricted use, distribution, and reproduction in any medium, provided the original work is properly cited.

\begin{abstract}
This article deals with an inverse problem of determining a linear source term in the multidimensional diffusion equation using the variational adjoint method. A variational identity connecting the known data with the unknown is established based on an adjoint problem, and a conditional uniqueness for the inverse source problem is proved by the approximate controllability to the adjoint problem under the condition that the unknowns can keep orders locally. Furthermore, a bilinear form is set forth also based on the variational identity and then a norm for the unknowns is well-defined by which a conditional Lipschitz stability is established.
\end{abstract}

\section{Introduction}

Air pollution and fog-haze problems have attracted much attention during the last decade in North China. There are some important aspects in the research of air pollution, such as the component and property of the air-pollutant, the accumulating process, and the migrating rule and the diffusion model. It is an effective method to analyze the transport and diffusion behaviors utilizing suitable advectiondiffusion equations (cf. [1-3], for instance). However, for reallife diffusion problems, some physical parameters are always unknown, or can not be measured directly, or can be obtained but expanding much cost, such as the diffusion coefficient, the initial distribution of the pollutant, and the physical/chemical source term. Therefore, it becomes feasible and necessary to put forward inverse problems using some additional data to determine those parameters with less cost in mathematics. An inverse problem arising in a diffusion equation belongs to inverse problems of parabolic type of partial differential equations.

By the literatures we have, there are quite a few of studies on inverse problems for parabolic equations since 1970s. As for general researches and summary, we refer to the monograph [4], and, for the research methods, we refer to [5-15] for the fixed point method based on the solution's expression of the forward problem, refer to [16-19] for the orthogonality method and energy estimates method, refer to [20-23] for utilizing the maximum principle, and refer to [24-28] for Carleman-type estimates method, and so forth. It is noted that stability analysis is still a trouble for inverse problems, especially for the construction of Lipschitz stability. By using classical estimates for parabolic problems in Hölder spaces, Hölder stability can be obtained based on the maximum principles and Sobolev embedding theorems; see also [4, 21, $22]$ and so forth. However, such method always needs more conditions for the initial boundary value functions, and it always involves complicated integral estimates. On the other hand, the variational identity method, also known as monotonicity method or the adjoint method, see, for example, [29$35]$, has been applied to parameter identification problems in the parabolic equations, by which uniqueness results can be proved using approximate controllability for the adjoint problems based on integral identities. The author discussed some inverse source problems for parabolic equations in $1 \mathrm{D}$ case (see [36-38] for instance) and gave conditional stability estimates for determining the source term or source coefficient using the variational identity method. Recently, an inverse problem of determining the first-order coefficient in an advection-dispersion equation in $2 \mathrm{D} / 3 \mathrm{D}$ case using the final observations was discussed also using the variational adjoint 
method [39], but the research domain is limited to be regular and the unknown should keep a form of variables separable, and the solution to the adjoint problem should have an explicit expression in order to prove the Lipschitz stability of the inverse problem.

In this paper we continue to deal with the inverse source problem for the multidimensional diffusion equation in a bounded open domain in $\mathbf{R}^{d}(d \geq 1)$. Here the inverse source problem is to determine the space-dependent source coefficient using the flux at partial boundary. A variational identity connecting the known data with the unknown source coefficient is established based on a suitable adjoint problem, and the uniqueness of the inverse problem is proved by the approximate controllability to the adjoint problem under the condition that the unknowns can keep orders locally. Furthermore, a bilinear form is set forth by the variational identity and then a norm for the unknowns is well-defined by which a conditional Lipschitz stability is constructed. It is noted that the maximum-minimum principle for parabolic equations is employed to keep sign of the solution to the adjoint problem instead of utilizing the explicit expression of the solution to the adjoint problem in the proof of the stability. Such kind of research approach can give more wide applications of the variational adjoint method to study well-posedness of inverse problems arising in partial differential equations.

\section{The Forward Problem and the Inverse Source Problem}

2.1. The Forward Problem. Let $\Omega \subset R^{d}$ and $T>0$, denote $\Omega_{T}=\Omega \times(0, T)$, and let $\Gamma$ be the smooth boundary of $\Omega$. Consider the multidimensional diffusion equation:

$$
u_{t}-D \Delta u=s(x, t), \quad(x, t) \in \Omega_{T},
$$

where $u=u(x, t)$ denotes the concentration of the pollutant at the space point $x$ and the time $t, \Delta$ is the Laplace operator, $D>0$ is the diffusion coefficient, and $s(x, t)$ is a linear source term. Generally speaking, the source term has a variables separable form: $s(x, t)=f(x) \lambda(t)$, where $\lambda(t)>0$ is a timedependent attenuation factor and $f(x)$ is a space-dependent source magnitude.

The initial boundary value conditions are given as follows:

$$
\begin{aligned}
u(x, 0) & =u_{0}(x), \quad x \in \bar{\Omega}, \\
\left.u\right|_{\Gamma} & =g(x, t), \quad x \in \Gamma, \quad 0 \leq t \leq T .
\end{aligned}
$$

If the model parameters and the initial boundary value functions in the forward problem (1)-(2) are all known and satisfy suitable consistency conditions, then it is a well-posed deterministic problem by the theory of parabolic equations, and there exists a unique solution on $\Omega_{T}$. Here we omit some related assertions on the forward problem (1)-(2) and focus on the inverse source problem given in the following.

2.2. The Inverse Source Problem. Suppose that there occurs some polluting phenomenon in a bounded region, and the pollutant is mainly produced by some continuous source distributed in the region. If the source term can not be measured directly, an inverse problem is encountered. Let the timedependent factor $\lambda(t)$ in the source term $s(x, t)=\lambda(t) f(x)$ be known; we are to determine the space-dependent source magnitude function $f(x)$ using the additional flux data measured at the partial boundary of $\Gamma_{1} \subset \Gamma$. That is, we have the additional condition:

$$
\left.\frac{\partial u}{\partial v}\right|_{\Gamma_{1}}=h(x, t), \quad(x, t) \in \Gamma_{1} \times[0, T],
$$

where $v$ denotes the normal outward vector at the boundary $\Gamma_{1}$. Thus an inverse source problem is formulated by the problem (1)-(2) together with the overposed information (3).

Denote $\Gamma_{1}^{T}=\Gamma_{1} \times[0, T]$, and let $S_{E}=\left\{f \in L^{2}(\Omega):\|f\|_{2} \leq\right.$ $E\}$ be an admissible set for the unknowns, where $E>0$ is a positive constant. For any given $f \in S_{E}$, there is a unique solution to the forward problem, denoted by $u(f)(x, t)$. Together with the additional information, we define a mapping $G: L_{2}(\Omega) \rightarrow L_{2}\left(\Gamma_{1}^{T}\right)$ :

$$
G[f]:=\left.\frac{\partial u}{\partial \nu}(f)\right|_{\Gamma_{1}}=h(x, t), \quad(x, t) \in \Gamma_{1}^{T} .
$$

Then the inverse problem (1)-(3) can be transformed to the problem of solving the operator equation (4). From the viewpoint of numerics, solving the inverse problem is reduced to solve the minimization problem with regularization strategy

$$
\min _{f \in S_{E}}\left\{\|G[f]-h\|_{2}^{2}+\alpha\|f\|_{2}^{2}\right\}
$$

where $\alpha>0$ is the regularization parameter. This paper is devoted to the well-posedness of the inverse source problem; numerical inversions will be presented in another occasion. Therefore, the following lemma on the approximate controllability to the heat equation is useful.

Lemma 1 (see [40]). Let $\Omega \subset \mathbf{R}^{d}$ be a bounded open subset with piecewise-smooth boundary $\partial \Omega$ having finite $d-1$ dimensional volume, $W$ be the set of all admissible boundary functions $w: \partial \Omega \times(0, T) \rightarrow \mathbf{R}$, and $\mathscr{A}$ be any nonempty set of $\partial \Omega \times(0, T)$. For $w \in W$, let $u(\cdot)=u(\cdot ; w):(0, T) \rightarrow L^{2}(\Omega)$ be the solution to the following initial boundary value problem:

$$
\begin{aligned}
u_{t}-\Delta u & =0, \\
\left.u\right|_{t=0} & =0, \\
\left.u\right|_{\partial \Omega} & =w .
\end{aligned}
$$

Then for any given $\psi \in L^{2}(\Omega)$ and $\varepsilon>0$, there exists a function $w^{*}$, continuous in $\mathscr{A}$, such that

$$
\left\|u\left(T ; w^{*}\right)-\psi\right\|_{L^{2}(\Omega)}<\varepsilon
$$

The assertion of this lemma is also valid to general parabolic equations, and it reveals that the final value of a homogeneous linear parabolic model can be approximately controlled by its boundary value. 


\section{Uniqueness of the Inverse Source Problem}

3.1. The Variational Identity. We give a variational identity connecting the known data with the unknown source function based on a suitable adjoint problem.

Theorem 2. Suppose that the initial and boundary value functions $u_{0}(x)$ and $g(x, t)$ are given and fixed here. Let $f_{i}(x) \in$ $L^{2}(\Omega)(i=1,2)$ and $u_{i}=u\left(f_{i}\right)(x, t)$ be the solutions to the forward problem (1)-(2) corresponding to $f_{i}(x)$ and $G\left[f_{i}\right]=$ $h_{i}(x, t)(i=1,2)$ be the additional flux data measured at $\Gamma_{1}$. Then there holds

$$
\begin{aligned}
& \int_{\Omega_{T}} \lambda(t)\left(f_{1}-f_{2}\right) \varphi(\omega)(x, t) d x d t \\
& \quad=-D \int_{\Gamma_{1}^{T}}\left(G\left[f_{1}\right]-G\left[f_{2}\right]\right) \omega(x, t) d S d t,
\end{aligned}
$$

where $\varphi(\omega)=\varphi(\omega)(x, t)$ satisfies the adjoint problem

$$
\begin{aligned}
\varphi_{t}+D \Delta \varphi & =0, \quad(x, t) \in \Omega_{T}, \\
\left.\varphi(x, t)\right|_{t=T} & =0, \quad x \in \bar{\Omega}, \\
\left.\varphi(x, t)\right|_{\Gamma} & = \begin{cases}\omega(x, t), & x \in \Gamma_{1}, t \in[0, T] ; \\
0, & x \in \Gamma \backslash \Gamma_{1}, t \in[0, T] .\end{cases}
\end{aligned}
$$

And $\omega=\omega(x, t)$ is a controllable boundary input.

Proof. Denote $U=u_{1}-u_{2}$; we have

$$
\begin{aligned}
U_{t}-D \Delta U & =\lambda(t)\left(f_{1}-f_{2}\right), \\
\left.U\right|_{t=0} & =0, \\
\left.U\right|_{\Gamma} & =0,
\end{aligned}
$$

and the additional information

$$
\left.\frac{\partial U}{\partial v}\right|_{\Gamma_{1}}=h_{1}-h_{2}
$$

By smooth test function $\varphi=\varphi(x, t)$, multiplying the two sides of the first equation in (10), and integrating on $\Omega_{T}$, there holds

$$
\begin{aligned}
& \int_{\Omega_{T}}\left(U_{t}-D \Delta U\right) \varphi d x d t \\
& \quad=\int_{\Omega_{T}} \lambda(t)\left(f_{1}-f_{2}\right) \varphi d x d t .
\end{aligned}
$$

Noting the homogeneous boundary conditions in (10), we have by integration by parts for the left-hand side of (12)

$$
\begin{gathered}
-\int_{\Omega_{T}} U\left[\varphi_{t}+D \Delta \varphi\right] d x d t+\int_{\Omega} U(x, T) \varphi(x, T) d x \\
-D \int_{0}^{T} \int_{\Gamma} \varphi \frac{\partial U}{\partial \nu} d S d t \\
=\int_{\Omega_{T}} \lambda(t)\left(f_{1}-f_{2}\right)(x) \varphi(x, t) d x d t .
\end{gathered}
$$

Let $\varphi=\varphi(x, t)$ be the solution to the adjoint problem (9); then it follows that (8) is valid by (13) together with the condition (11). Since the adjoint problem (9) is uniquely determined by its boundary input $\omega(x, t)$, we denote its solution as $\varphi=\varphi(\omega)(x, t)$. The proof is over.

3.2. Conditional Uniqueness. Uniqueness is an important aspect in the research of inverse problems. With the help of the variational identity (8) and Lemma 1 , we will prove a conditional uniqueness for the inverse source problem given above. The required condition is that the unknown function should keep its sign in a small region of the considered domain.

Theorem 3. Under the conditions of Theorem 2, also assume that $\lambda(t) \in C([0, T])$ and $\lambda(t)>0$ for $t \in[0, T]$. If $h_{1}=h_{2}$ on $\Gamma_{1}^{T}$, then there is no positive measurable subdomain of $\Omega$ where $f_{1}-f_{2}$ is of one sign.

Proof. By the assumption of $h_{1}=h_{2}$ on $\Gamma_{1}^{T}$, we have by identity (8)

$$
\int_{\Omega_{T}} \lambda(t)\left(f_{1}-f_{2}\right) \varphi(\omega)(x, t) d x d t=0 .
$$

Suppose that there is a subdomain $\Omega_{0} \subset \Omega$ with positive measure such that $f_{1}-f_{2}$ keeps one sign. Let $\left.\left(f_{1}-f_{2}\right)\right|_{\Omega_{0}} \geq 0$ for convenience. We will deduce a contradiction with (14) in the following.

Denote

$$
I=\int_{\Omega}\left(f_{1}-f_{2}\right)\left[\int_{0}^{T} \lambda(t) \varphi(\omega)(x, t) d t\right] d x .
$$

Take a partition of the time interval $[0, T]$ :

$$
0=t_{0}<t_{1}<\cdots<t_{N-1}<t_{N}=T,
$$

and denote $\Delta t=t_{i}-t_{i-1}, i=1,2, \ldots, N$; we have

$$
\begin{aligned}
I= & \int_{\Omega}\left(f_{1}-f_{2}\right)\left[\sum_{i=0}^{N-1} \lambda\left(t_{i}\right) \varphi(\omega)\left(x, t_{i}\right) \Delta t\right] d x \\
& +M_{1}(\Delta t)^{2}
\end{aligned}
$$

where $M_{1}(\Delta t)^{2}$ denotes the truncated error and $M_{1}>0$ is a positive constant. Noting the condition $\left.\varphi(\omega)\right|_{t=T}=0$, here the term of $i=N$ is omitted.

By the assumption, there exists a nonnegative integrable function $\xi(x)$ on $\Omega$ given by

$$
\xi(x)= \begin{cases}r(x), & \Omega_{0} ; \\ 0, & \Omega \backslash \Omega_{0},\end{cases}
$$

where $r(x)$ is a smooth positive function on $\Omega_{0}$. Thus we get

$$
\begin{aligned}
& \int_{\Omega}\left(f_{1}-f_{2}\right)(x) \xi(x) d x=\int_{\Omega_{0}}\left(f_{1}-f_{2}\right)(x) r(x) d x \\
& \quad=: M>0 .
\end{aligned}
$$


In addition, we define a series of integrable functions on $\Omega$ by the assumption on $\lambda(t)$ :

$$
\begin{aligned}
& V_{i}(x)=\frac{\xi(x)}{\lambda\left(t_{i}\right)}= \begin{cases}\frac{r(x)}{\lambda\left(t_{i}\right)}, & x \in \Omega_{0}, \\
0, & x \in \Omega \backslash \Omega_{0},\end{cases} \\
& i=0,1, \ldots, N-1 \text {. }
\end{aligned}
$$

Noting that the adjoint problem (9) is a well-posed backward problem and the initial state is zero at $t=T$, we deduce that its solution $\varphi(\omega)(x, t)$ at $t=t_{N-1}$ can be approximated to any objective function $V_{N-1}(x) \in L^{2}(\Omega)$ by Lemma 1 by suitably choosing the boundary input $\omega(x, t)$. Subsequently, there exists a boundary input on each subinterval $\left[t_{i-1}, t_{i}\right]$ such that for any $\varepsilon>0$ we have

$$
\begin{array}{r}
\left\|\varphi(\omega)\left(x, t_{i}\right)-V_{i}(x)\right\|_{L_{2}(\Omega)} \leqslant \frac{M \varepsilon}{\|\lambda\|_{\infty}\left\|f_{1}-f_{2}\right\|_{L^{2}(\Omega)}} \\
i=N-1, \ldots, 0 .
\end{array}
$$

Based on (17) and (19), utilizing the basic inequality and noting $\sum_{i=0}^{N-1}|\Delta t|=t_{N-1}$, we get

$$
\begin{aligned}
|I| & \geq\left|\int_{\Omega}\left(f_{1}-f_{2}\right)\left[\sum_{i=0}^{N-1} \lambda\left(t_{i}\right) \varphi(\omega)\left(x, t_{i}\right) \Delta t\right] d x\right| \\
& -M_{1}(\Delta t)^{2}=\mid \int_{\Omega}\left(f_{1}-f_{2}\right) \\
& \cdot\left[\sum_{i=0}^{N-1}\left(\lambda\left(t_{i}\right) \varphi(\omega)\left(x, t_{i}\right)-\xi(x)+\xi(x)\right) \Delta t\right] d x \mid \\
& -M_{1}(\Delta t)^{2} \geq M t_{N-1}-\mid \int_{\Omega}\left(f_{1}-f_{2}\right) \\
& \cdot\left[\sum_{i=0}^{N-1}\left(\lambda\left(t_{i}\right) \varphi(\omega)\left(x, t_{i}\right)-\xi(x)\right) \Delta t\right] d x \mid \\
& -M_{1}(\Delta t)^{2} .
\end{aligned}
$$

Thanks to $\xi(x)=\lambda\left(t_{i}\right) V_{i}(x)$, there holds the estimate for the center term of the right-hand side in (22) by (21) and CauchySchwartz inequality

$$
\begin{aligned}
& \mid \int_{\Omega}\left(f_{1}-f_{2}\right) \\
& \quad \cdot\left[\sum_{i=0}^{N-1}\left(\lambda\left(t_{i}\right) \varphi(\omega)\left(x, t_{i}\right)-\xi(x)\right) \Delta t\right] d x \mid \\
& \quad \leq\|\lambda\|_{\infty} \\
& \quad \cdot \sum_{i=0}^{N-1} \int_{\Omega}\left|\left(f_{1}-f_{2}\right)\left[\varphi(\omega)\left(x, t_{i}\right)-V_{i}(x)\right]\right| d x|\Delta t|
\end{aligned}
$$

$$
\begin{aligned}
& \leq\|\lambda\|_{\infty}\left\|f_{1}-f_{2}\right\|_{2} \sum_{i=0}^{N-1}\left\|\varphi(\omega)\left(x, t_{i}\right)-V_{i}(x)\right\|_{2}|\Delta t| \\
& \leq M \varepsilon t_{N-1} .
\end{aligned}
$$

Henceforth, we have

$$
|I| \geqslant(1-\varepsilon) M t_{N-1}-M_{1}(\Delta t)^{2} .
$$

It follows that there must be $|I|>0$ as long as $\varepsilon$ and $|\Delta t|$ are small enough, which is a contradiction with (14). The proof is completed.

\section{Conditional Lipschitz Stability}

Firstly we give a basic assertion.

Lemma 4. Let $\Omega$ be bounded measurable domain. Suppose that $f(x) \in L^{2}(\Omega)$ and $f(x) \geq 0$ for $x \in \Omega$; then $\int_{\Omega} f(x) d x=$ 0 implies that $f(x)=0$ a.e. $x \in \Omega$.

With a complete method as that used in Theorem 2, we can prove the following assertion.

Theorem 5. Let $\lambda(t) \in L^{2}(0, T), f_{i}(x), u_{0}^{i}(x) \in L^{2}(\Omega)$, $g_{i}(x, t) \in L^{2}\left(\Gamma_{T}\right)(i=1,2)$, and $u_{i}=u\left(f_{i}\right)(x, t)$ be the solutions of the forward problem (1)-(2) corresponding to $u_{0}^{i}(x)$, $g_{i}(x, t)$, and $f_{i}(x), i=1,2$, and $\left.\left(\partial u_{i} / \partial v\right)\right|_{\Gamma_{1}^{T}}=h_{i}(x, t)(i=$ $1,2)$ be the additional flux data. Then there holds

$$
\begin{gathered}
\int_{\Omega_{T}} \lambda(t)\left(f_{1}-f_{2}\right) \varphi(\omega)(x, t) d x d t \\
=-D \int_{\Gamma_{1}^{T}}\left(h_{1}-h_{2}\right) \omega(x, t) d S d t \\
\quad+D \int_{\Gamma^{T}}\left(g_{1}-g_{2}\right) \frac{\partial \varphi}{\partial \nu}(x, t) d S d t \\
-\int_{\Omega}\left(u_{0}^{1}-u_{0}^{2}\right) \varphi(x, 0) d x,
\end{gathered}
$$

where $\varphi(\omega)=\varphi(\omega)(x, t)$ is also the solution to the adjoint problem (9).

For construction of stability for the inverse source problem, we need extra conditions for the known and unknown data functions. For the unknown source coefficient function $f \in S_{E}$, we further assume that $f(x) \geq 0$ and $f \neq \equiv$ for $x \in \Omega$. In addition we assume that the attenuation factor $\lambda(t)$ is continuous and positive on $[0, T]$ and $\|\lambda\|_{\infty} \geq \delta_{0}>0$; here $\delta_{0}$ is a positive constant. Now, based on the variational identity (8), we define a functional on the source function $f(x)$ and the controllable input $\omega(x, t)$ :

$$
\mathscr{B}(f, \omega)=\int_{\Omega_{T}} \lambda(t) f(x) \varphi(\omega)(x, t) d x d t,
$$

where $\varphi(\omega)(x, t)$ is the solution to the adjoint problem (9) which only depends upon the boundary input $\omega=\omega(x, t)$ for $(x, t) \in \Gamma_{1}^{T}$. 
It is not difficult to show that the bilinear functional $\mathscr{B}(f, \omega)$ is bounded on $f$ and $\omega$ in $L^{2}$ norm. Then we define a norm on $f$ which is called $\mathscr{B}$-norm given by

$$
\|f\|_{\mathscr{B}}:=\sup _{\omega \geq 0, \omega \equiv 0} \frac{|\mathscr{B}(f, \omega)|}{\|\lambda\|_{\infty}\|\omega\|_{2}} .
$$

We need to prove that the norm given by (27) is welldefined. Obviously, $\mathscr{B}(f, \omega)$ is nonnegative and satisfies the absolute homogeneity and triangle inequality due to the linearity and additivity of the integration. We now prove that if $\|f\|_{\mathscr{B}}=0$, then there must be $f=0$ a.e. $x \in \Omega$ in the case of $\omega \geq 0$ and $\omega \neq \equiv$.

In fact, assume that $\|f\|_{\mathscr{B}}=0$; that is, there holds

$$
\left|\int_{\Omega_{T}} \lambda(t) f(x) \varphi(\omega)(x, t) d x d t\right|=0
$$

By the assumptions that $\omega \geq 0$ for $(x, t) \in \Gamma^{T}$ we deduce that the solution $\varphi=\varphi(\omega)$ to the adjoint problem (9) takes nonnegative values by the maximum-minimum principle for the parabolic equations (cf. [41], for instance). Together with $f(x) \geq 0$ and $\lambda(t) \geq \delta_{0}>0$ it follows that the integrated function in (28) takes nonnegative values; that is, we get

$$
\int_{\Omega_{T}} \lambda(t) f(x) \varphi(\omega)(x, t) d x d t=0
$$

Noting the assumption on the attenuation factor $\lambda(t)$, there must be $f=0$ a.e. $x \in \Omega$ or $\varphi(\omega)=0$ a.e. $(x, t) \in \Omega_{T}$ by Lemma 4 . Since the boundary input satisfies the condition $\omega \geq 0$ and $\omega \neq 0$, there holds $\varphi(\omega) \geq 0$ a.e. $(x, t) \in \Omega_{T}$ also by the maximum-minimum principle. So there must be $f=0$ a.e. $x \in \Omega$. Thus the norm given by (27) is well-defined.

Theorem 6. Under the conditions of Theorem 5, let $f \in S_{E}$ and take nonnegative values on $x \in \Omega$, and let $\lambda(t)$ be continuous and positive on $[0, T]$ and $\|\lambda\|_{\infty} \geq \delta_{0}>0$. Then there exists a positive constant $c=c\left(\Omega_{T}, D, \lambda(t), \omega\right)$ such that

$$
\begin{aligned}
& \left\|f_{1}-f_{2}\right\|_{\mathscr{B}} \leq c\left(\left\|h_{1}-h_{2}\right\|_{L^{2}\left(\Gamma_{1}^{T}\right)}+\left\|g_{1}-g_{2}\right\|_{L^{2}\left(\Gamma^{T}\right)}\right. \\
& \left.\quad+\left\|u_{0}^{1}-u_{0}^{2}\right\|_{L^{2}(\Omega)}\right),
\end{aligned}
$$

where the norm $\|\cdot\|_{\mathscr{B}}$ is defined by (27).

Proof. By the variational identity (25) and the norm definition (27), utilizing Cauchy-Schwartz inequality, we have

$$
\begin{gathered}
\left\|f_{1}-f_{2}\right\|_{\mathscr{B}} \leq \frac{1}{\|\lambda\|_{\infty}} \cdot\left\{D\left\|h_{1}-h_{2}\right\|_{L^{2}\left(\Gamma_{1}^{T}\right)}\right. \\
+D\left\|g_{1}-g_{2}\right\|_{L^{2}\left(\Gamma^{T}\right)} \sup _{\omega \geq 0, \omega \neq 0} \frac{\|(\partial \varphi / \partial v)(x, t)\|_{2}}{\|\omega\|_{2}} \\
\left.+\left\|u_{0}^{1}-u_{0}^{2}\right\|_{L^{2}(\Omega)} \sup _{\omega \geq 0, \omega \neq 0} \frac{\|\varphi(x, 0)\|_{2}}{\|\omega\|_{2}}\right\} .
\end{gathered}
$$

By general regularity theory of parabolic equations with the initial boundary value conditions, we know that, for $\omega \epsilon$ $L^{2}\left(\Gamma_{1}^{T}\right)$, there exists a constant $c_{1}=c_{1}\left(D, \Omega_{T}\right)$ such that

$$
\|\varphi(x, 0)\|_{2},\left\|\frac{\partial \varphi}{\partial \nu}(x, t)\right\|_{2} \leq c_{1}\|\omega\|_{2} .
$$

Henceforth we get

$$
\begin{aligned}
& \left\|f_{1}-f_{2}\right\|_{\mathscr{B}} \\
& \leq \frac{1}{\|\lambda\|_{\infty}} \\
& \quad \cdot\left\{D\left\|h_{1}-h_{2}\right\|_{2}+D c_{1}\left\|g_{1}-g_{2}\right\|_{2}+c_{1}\left\|u_{0}^{1}-u_{0}^{2}\right\|_{2}\right\} .
\end{aligned}
$$

Noting (31) and the condition $\|\lambda\|_{\infty} \geq \delta_{0}>0$, by setting

$$
c=\frac{1}{\delta_{0}} \max \left\{D, D c_{1}, c_{1}\right\}
$$

it follows that the assertion of this theorem is valid.

\section{Conclusions}

An inverse problem of determining a space-dependent source coefficient in the multidimensional diffusion equation using the variational adjoint method is investigated. A variational identity connecting the known data with the unknown is established based on an adjoint problem, and uniqueness for the inverse source problem is proved by the approximate controllability to the adjoint problem under suitable conditions. This is the conditional uniqueness. Also, based on the variational identity, a bilinear form is set forth by which a norm for the unknown source is well-defined, and then a Lipschitz stability can be proved if the unknown source is of one sign. Such method can be generalized to study the Lipschitz stability for other kinds of inverse coefficient problems arising in partial differential equations.

\section{Conflicts of Interest}

The authors declare that there are no conflicts of interest regarding the publication of this paper.

\section{Acknowledgments}

This work is partially supported by the National Natural Science Foundation of China (nos. 11371231, 11071148).

\section{References}

[1] G. S. Li and D. Yao, The inversion Problems of Source Terms and Applications, Science Press, Beijing, 2014.

[2] N.-Z. Sun, Mathematical modeling of groundwater pollution, Springer-Verlag, New York, 1996.

[3] C. M. Zheng and G. D. Bennett, "Applied Contaminant Transport Modeling," John Wiley Sons, New York, NY, USA, 2002.

[4] V. Isakov, Inverse Problems for Partial Differential Equations, vol. 127 of Applied Mathematical Sciences, Springer, New York, NY, USA, 1998. 
[5] J. R. Cannon, The One-Dimensional Heat Equation, AddisonWesley, Reading, Mass, USA, 1984.

[6] J. R. Cannon and H. Yin, "A uniqueness theorem for a class of nonlinear parabolic inverse problems," Inverse Problems, vol. 4, no. 2, pp. 411-416, 1988.

[7] J. R. Cannon and S. Perez-Esteva, "Uniqueness and stability of 3D heat sources," Inverse Problems, vol. 7, no. 1, pp. 57-62, 1991.

[8] P. DuChateau and W. Rundell, "Unicity in an inverse problem for an unknown reaction term in a reaction-diffusion equation," Journal of Differential Equations, vol. 59, no. 2, pp. 155-164, 1985.

[9] S. Gatti, "An existence result for an inverse problem for a quasilinear parabolic equation," Inverse Problems, vol. 14, no. 1, pp. 53-65, 1998.

[10] L. Gongsheng, M. Yichen, and L. Kaitai, "An inverse parabolic problem for nonlinear source term with nonlinear boundary conditions," Journal of Inverse and Ill-Posed Problems, vol. 11, no. 4, pp. 371-387, 2003.

[11] A. Nanda and P. C. Das, "Determination of the source term in the heat conduction equation," Inverse Problems, vol. 12, no. 3, pp. 325-339, 1996.

[12] M. Pilant and W. Rundell, "Fixed point methods for a nonlinear parabolic inverse coefficient problem," Communications in Partial Differential Equations, vol. 13, no. 4, pp. 469-493, 1988.

[13] M. Pilant and W. Rundell, "A method for identifying nonlinear terms in parabolic initial-boundary value problems," Advances in Water Resources, vol. 14, no. 2, pp. 83-88, 1991.

[14] W. Rundell, "The determination of a parabolic equation from initial and final data," Proceedings of the American Mathematical Society, vol. 99, no. 4, pp. 637-642, 1987.

[15] A. Zeghal, "Existence results for inverse problems associated with a nonlinear parabolic equation," Journal of Mathematical Analysis and Applications, vol. 272, no. 1, pp. 240-248, 2002.

[16] I. Bushuyev, "Global uniqueness for inverse parabolic problems with final observation," Inverse Problems, vol. 11, no. 4, article no. 001, pp. L11-L16, 1995.

[17] V. Isakov, Inverse Source Problems, vol. 34 of Mathematical Surveys and Monographs, American Mathematical Society, Providence, RI, USA, 1990.

[18] E. G. Savateev and R. Riganti, "Inverse problem for the nonlinear heat equation with the final overdetermination," Mathematical and Computer Modelling, vol. 22, no. 1, pp. 29-43, 1995.

[19] M. Yamamoto, "Conditional stability in determination of force terms of heat equations in a rectangle," Mathematical and Computer Modelling, vol. 18, no. 1, pp. 79-88, 1993.

[20] M. Choulli and M. Yamamoto, "Conditional stability in determining a heat source," Journal of Inverse and Ill-Posed Problems, vol. 12, no. 3, pp. 233-243, 2004.

[21] V. Isakov, "Inverse parabolic problems with the final overdetermination," Communications on Pure and Applied Mathematics, vol. 44, no. 2, pp. 185-209, 1991.

[22] V. Isakov, "Some inverse problems for the diffusion equation," Inverse Problems, vol. 15, no. 1, pp. 3-10, 1999.

[23] W. Rundell, "Determination of an unknown nonhomogeneous term in a linear partial differential equation from overspecified boundary data," Applicable Analysis, vol. 10, no. 3, pp. 231-242, 1980.

[24] V. Isakov, "Carleman type estimates in an anisotropic case and applications," Journal of Differential Equations, vol. 105, no. 2, pp. 217-238, 1993.

[25] M. V. Klibanov, "Inverse problems and Carleman estimates," Inverse Problems, vol. 8, no. 4, article no. 009, pp. 575-596, 1992.
[26] M. V. Klibanov and A. Timonov, Carleman estimates for coefficient inverse problems and numerical applications, Inverse and Ill-posed Problems Series, VSP, Utrecht, 2004.

[27] M. Machida and M. Yamamoto, "Global Lipschitz stability in determining coefficients of the radiative transport equation," Inverse Problems, vol. 30, no. 3, Article ID 035010, 2014.

[28] M. Yamamoto, "Carleman estimates for parabolic equations and applications," Inverse Problems, vol. 25, no. 12, Article ID 123013, 123013, 75 pages, 2009.

[29] J. R. Cannon and P. DuChateau, "Structural identification of an unknown source term in a heat equation," Inverse Problems, vol. 14, no. 3, pp. 535-551, 1998.

[30] P. DuChateau, "Monotonicity and invertibility of coefficient-todata mappings for parabolic inverse problems," SIAM Journal on Mathematical Analysis, vol. 26, no. 6, pp. 1473-1487, 1995.

[31] P. DuChateau, "An inverse problem for the hydraulic properties of porous media," SIAM Journal on Mathematical Analysis, vol. 28, no. 3, pp. 611-632, 1997.

[32] P. DuChateau, R. Thelwell, and G. Butters, "Analysis of an adjoint problem approach to the identification of an unknown diffusion coefficient," Inverse Problems, vol. 20, no. 2, pp. 601625, 2004.

[33] P. DuChateau, "An adjoint method for proving identifiability of coefficients in parabolic equations," Journal of Inverse and IllPosed Problems, vol. 21, no. 5, pp. 639-663, 2013.

[34] A. Hasanov, P. DuChateau, and B. Pektas, "An adjoint problem approach and coarse-fine mesh method for identification of the diffusion coefficient in a linear parabolic equation," Journal of Inverse and Ill-Posed Problems, vol. 14, no. 5, pp. 435-463, 2006.

[35] Y. H. Ou, A. Hasanov, and Z. H. Liu, "Inverse coefficient problems for nonlinear parabolic differential equations," Acta Mathematica Sinica, vol. 24, no. 10, pp. 1617-1624, 2008.

[36] L. Gongsheng and M. Yamamoto, "Stability analysis for determining a source term in a 1-D advection-dispersion equation," Journal of Inverse and Ill-Posed Problems, vol. 14, no. 2, pp. 147$155,2006$.

[37] G. Li, "Data compatibility and conditional stability for an inverse source problem in the heat equation," Applied Mathematics and Computation, vol. 173, no. 1, pp. 566-581, 2006.

[38] G. Li, D. Yao, and F. Yang, "An inverse problem of identifying source coefficient in solute transportation," Journal of Inverse and Ill-Posed Problems, vol. 16, no. 1, pp. 51-63, 2008.

[39] G. Li, X. Jia, and C. Sun, “A conditional Lipschitz stability for determining a space-dependent source coefficient in the $2 \mathrm{D} / 3 \mathrm{D}$ Advection-dispersion equation," Journal of Inverse and Ill-Posed Problems, vol. 25, no. 2, pp. 221-236, 2017.

[40] R. C. MacCamy, V. J. Mizel, and T. I. Seidman, "Approximate boundary controllability for the heat equation," Journal of Mathematical Analysis and Applications, vol. 23, pp. 699-703, 1968.

[41] R. P. Sperb, Maximum Principles and Their Applications, vol. 157 of Mathematics in Science and Engineering, Academic Press, New York, NY, USA, 1981. 


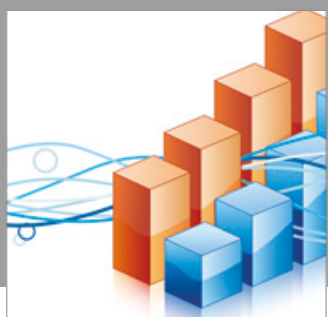

Advances in

Operations Research

vatersals

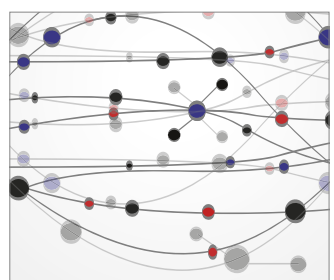

\section{The Scientific} World Journal
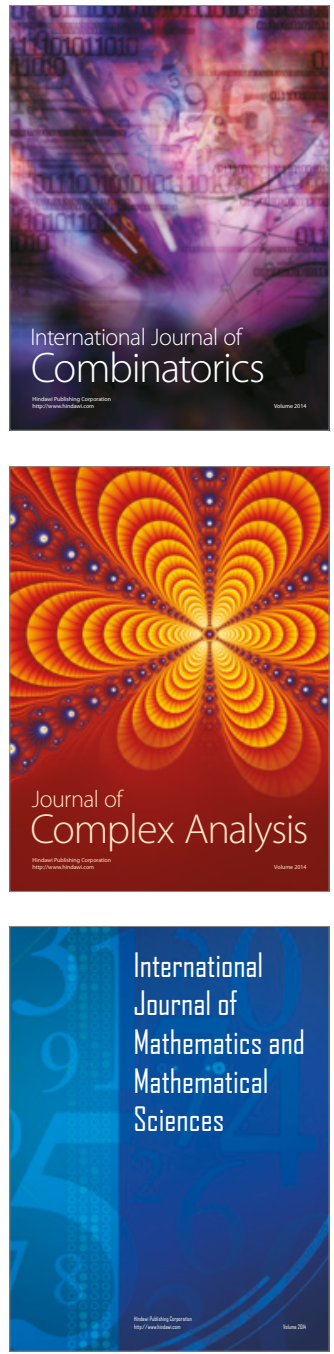
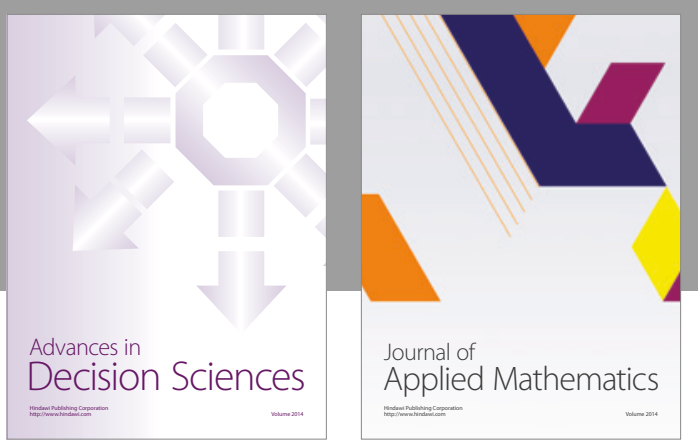

Algebra

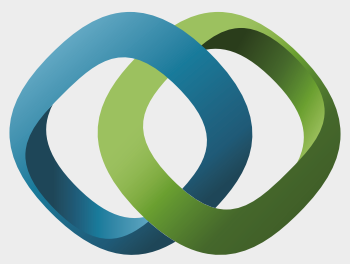

\section{Hindawi}

Submit your manuscripts at

https://www.hindawi.com
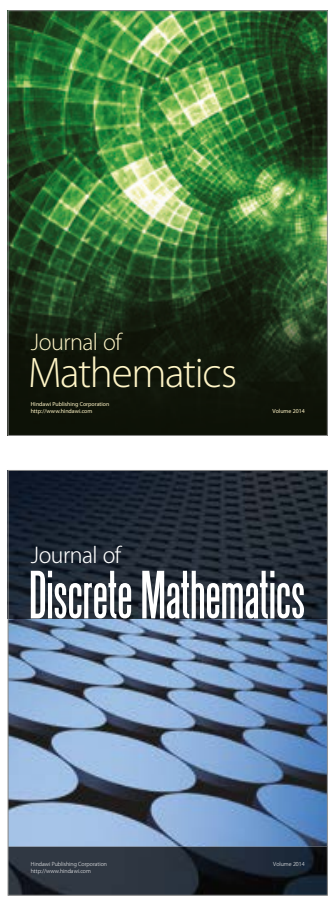

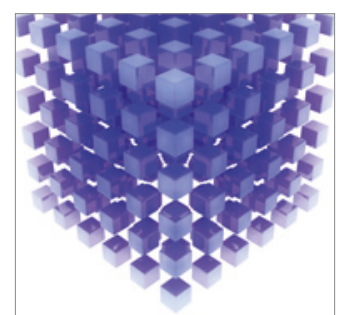

Mathematical Problems in Engineering
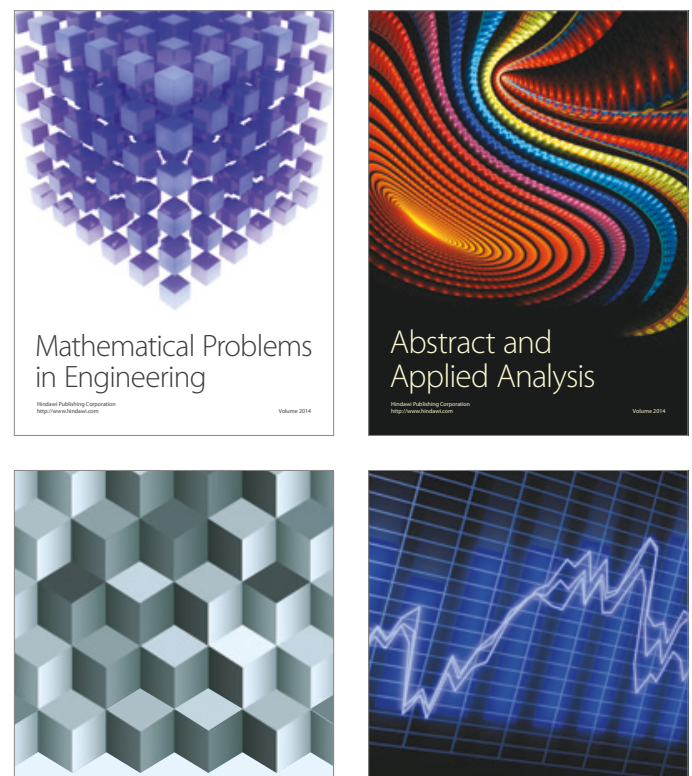

Journal of

Function Spaces

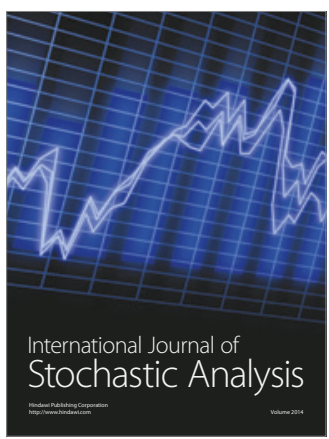

Probability and Statistics
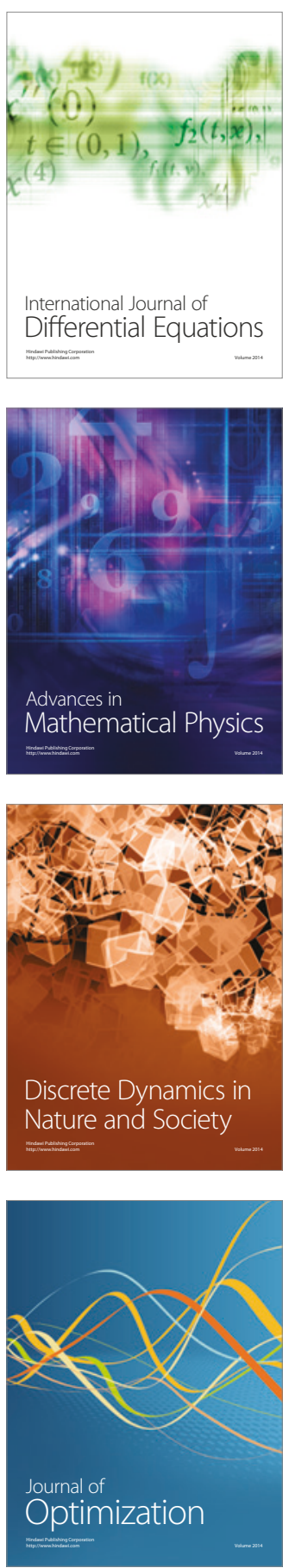